\title{
Biomechanical investigation of pelvic stability in developmental dysplasia of the hip: unilateral salter osteotomy versus one- stage bilateral salter osteotomy
}

\author{
Lang $\mathrm{Li}^{1 \dagger}$, Xiaodong Yang ${ }^{1 \dagger}$, Bo Song ${ }^{2 \dagger}$, Jun Jiang ${ }^{1}$, Lei Yang ${ }^{1}$ and Xueyang Tang ${ }^{1 *}$
}

\begin{abstract}
Background: Developmental dysplasia of the hip (DDH) is a common disease in infants and children, and the treatment of bilateral DDH remains controversial. This study aimed to evaluate the stability of one-stage bilateral Salter pelvic osteotomy for bilateral DDH in patients of walking age.

Methods: In total, nine child cadavers aged 2-6 years were included. A universal mechanical testing machine was used for stability test. We performed two different surgical procedures on the specimens: nine child cadavers underwent unilateral Salter pelvic osteotomy, and six child cadavers were randomly selected to undergo Salter pelvic osteotomy again to simulate one-stage bilateral Salter pelvic osteotomy. The stability of the bilateral sacroiliac joints, local stability of the operation area, ultimate load test, and axial stiffness were evaluated.

Results: Both unilateral and bilateral Salter osteotomy could destroy the integrity of the pelvic ring and increase the risk of pelvic instability. In this study, compared with unilateral Salter osteotomy, bilateral Salter osteotomy had similar pelvic stability, and there was no significant difference between unilateral and bilateral Salter osteotomy in sacroiliac joint stability $(p>0.05)$, local stability $(p=0.763)$, ultimate load $(p=0.328)$, and axial stiffness $(p=0.480)$.

Conclusions: One-stage bilateral Salter pelvic osteotomy as a potential surgical method is viable and stable for children with bilateral DDH.
\end{abstract}

Keywords: Developmental dysplasia of the hip, Salter osteotomy, One-stage, Unilateral

\section{Introduction}

Developmental dysplasia of the hip (DDH) is a common disease in infants and children, and is based on congenital acetabular dysplasia. The incidence of DDH on the left side is $60 \%, 20 \%$ on the right side, and $20 \%$ bilaterally [1]. Many infants and children do not receive conservative treatment timeously which leads to abnormal gait/

\footnotetext{
* Correspondence: xueyangtwch@163.com

${ }^{\dagger}$ Lang Li, Xiaodong Yang, and Bo Song contributed equally to this work. 1 Department of Pediatric Surgery, West China Hospital, Sichuan University, Chengdu 610041, Sichuan, China

Full list of author information is available at the end of the article
}

osteoarthritis. As a result, DDH is very common in pediatric orthopedics in developing and low-income countries [2]. Acetabular dysplasia often worsens if the diagnosis is delayed and can cause varying degrees of hip dislocation requiring different treatments for DDH [3-5]. Patients who are diagnosed early can be successfully treated with conservative methods such as a Pavlik harness, but surgical treatment becomes inevitable for most patients with a delayed diagnosis [6].

In 1961, Salter first described open reduction and pelvic osteotomy for DDH. Salter osteotomy has subsequently become a popular treatment option for 
walking-age children with DDH [7]. Salter pelvic osteotomy changes the direction of the acetabulum by cutting the ilium completely and using the pubic symphysis as a hinge to rotate the distal end of the osteotomy bone. This increases accommodation of the dislocated femoral head to achieve central reduction $[7,8]$. Salter pelvic osteotomy involves cutting the ilium followed by fixation with Kirschner wires; this can affect the stability of the pelvis, therefore some surgeons suggest that patients with bilateral DDH should receive successive Salter osteotomy because of the high risk of a poor Severin grading [9]. And Salter also thought one-stage bilateral Salter pelvic osteotomy to be contraindicated and recommended that the osteotomy on the second hip be performed two weeks after the first hip. Salter thought one-stage bilateral Salter pelvic osteotomy could result with loss of fixation [2]. However, some studies have also reported that one-stage bilateral pelvic osteotomy is reliable and economical [10-12]. One-stage bilateral osteotomy was viable and may be even better acetabular index than that of a unilateral osteotomy. However, there are currently no biomechanical experiment to explore and verify the pelvic stability of one-stage bilateral Salter pelvic osteotomy.

We are of the opinion that one-stage bilateral pelvic osteotomy with ilia cut bilaterally, will result in the same load on the left and right of the pelvic ring, thereby maintaining balance, and will not increase the risk of pelvic instability. In this study, we prepared pelvic specimens to evaluate the stability of one-stage bilateral Salter pelvic osteotomy in children aged 2 to 6 years.

\section{Materials and methods}

Pelvic specimens from four male and five female child cadavers aged 2-6years were obtained from the Department of Anatomy, West China of Medicine, Sichuan University. Approval was obtained from the Sichuan Province in accordance with the law for the protection of cadavers sampling of the Chinese Government.

\section{Sample preparation}

Nine child cadavers including five girls were unfrozen 1 day prior to experimrnt, and the pelvic specimens were dissected by a professional surgeon. The pelvic specimens were complete from the fifth lumbar vertebra to $10 \mathrm{~cm}$ of the proximal femur. The sacroiliac joint, hip joint, pubic symphysis, and related ligament were protected (Fig. 1a). Nine pelvic specimens were prepared for pelvic stability examination as follows:

The pelvis was fixed in a standing position, and stability was tested using a universal mechanical testing machine (AG-IS, Shimadzu, Japan). First, M1 and M3 were marked on the iliac side of the sacroiliac joint, respectively, and M2 was marked at the same level on the sacrum (Fig. 1b). The force added to the pelvis on the top of the fifth lumbar vertebra was from 0 to $400 \mathrm{~N}$ with a speed of $10 \mathrm{~N} / \mathrm{S}$ (Fig. 2a). Images were acquired every four seconds using a sophisticated camera (JHSM1400f, China), and displacements of M1-M3 were converted and processed by a computer. The principle of image displacement recognition method is as follows: the coordinate origin is directly above the M2 point; the $X$-axis direction represents the horizontal displacement to the right, and the $Y$-axis direction represents the
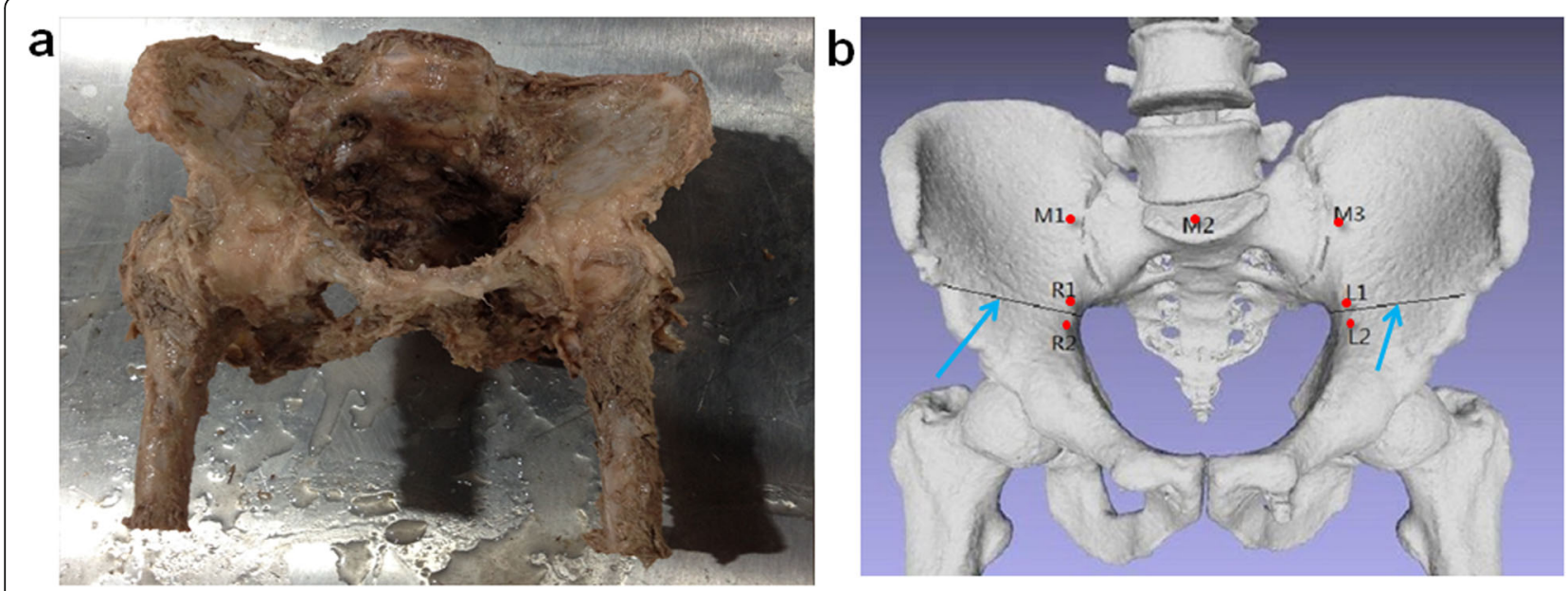

Fig. 1 Intact pelvis and marked point. a The intact pelvis before treated with different operation. b The marked point of M1, M2, M3, L1, L2, R1, and R2. M1 and M3 were marked on the iliac side of the sacroiliac joint, respectively, and M2 was marked at the same level on the sacrum. L1(R1) and L2(R2) were marked on both sides of Salter osteotomy, respectively. The blue arrow refers to the broken end of the osteotomy 


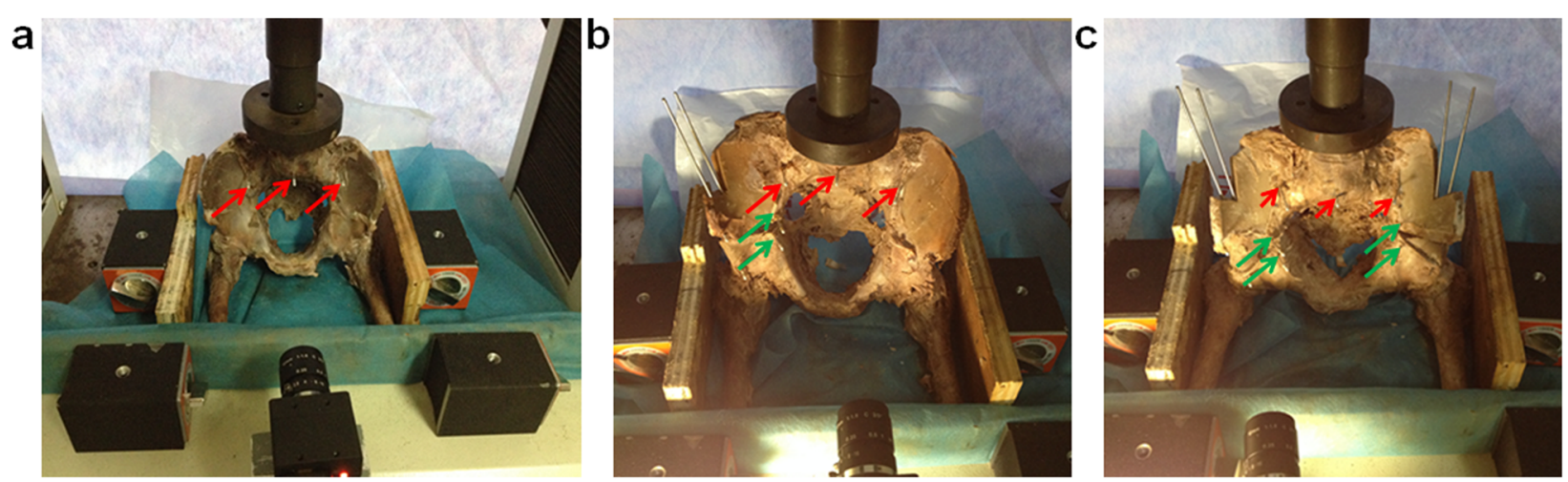

Fig. 2 Different osteotomy for pelvis and the displacement of marked point was acquired by sophisticated camera. a The intact pelvis. $\mathbf{b}$ The unilateral salter pelvic osteotomy. c One-stage bilateral Salter pelvic osteotomy. The red arrow refers to M1 or M2 or M3, and green arrow refers to $L 1(R 1)$ and $L 2(R 2)$

vertical displacement downwards. The positions of M1(x1, y1), M2(x2, y2), and M3(x3, y3) were obtained.

\section{Unilateral Salter pelvic osteotomy}

Unilateral Salter pelvic osteotomy was performed on nine pelvic specimens by a professional surgeon; left/ right ilium was chosen randomly with random numbers generated by computer. The implanted bone graft was taken from the ipsilateral ilium as an isosceles triangle and was the same basic size for all pelvic specimens (base is $2 \mathrm{~cm}$, apex angle is $30^{\circ}$ ). Two Kirschner wires were used to fix the implanted bone graft. After Salter osteotomy was performed, four points (L1, L2, L3, and L4) were marked (Fig. 1b). A force from 0 to $400 \mathrm{~N}$ with a speed of $10 \mathrm{~N} / \mathrm{S}$ was added to the pelvis on the top of the fifth lumbar vertebra. The displacements of M1, M2, M3, L1, L2, R1, and R2 were recorded and processed using the method described above (JHSM1400f, China) (Fig. 2b).

\section{Bilateral Salter pelvic osteotomy}

Six pelvic specimens that underwent unilateral Salter pelvic osteotomy were chosen randomly with a computer random number to receive another Salter pelvic osteotomy to simulate bilateral Salter pelvic osteotomy. The osteotomy was performed by a same professional surgeon as mentioned above. A force from 0 to $400 \mathrm{~N}$ with a speed of $10 \mathrm{~N} / \mathrm{S}$ was added to the pelvis. Seven points (M1, M2, M3, L1, L2, R1 and R2) were marked and all displacements were recorded (Fig. 2c).

\section{Axial stiffness and ultimate load test}

The vertical displacement of M2 (VD-M2) is an approximate representation of the displacement of the pelvic ring. In this study, we used 400 N/VD-M2 to determine axial stiffness. After the force test from $0 \mathrm{~N}$ to $400 \mathrm{~N}$, we performed an ultimate load test to evaluate the stability of the pelvic ring after different surgeries. A force from $0 \mathrm{~N}$ was added to the pelvis on the top of the fifth lumbar vertebra with a speed $10 \mathrm{~N} / \mathrm{S}$. Ultimate load was defined as the force that damaged the pelvis and caused the pelvic ring to become unstable.

\section{Statistical analysis}

All measurement data were presented as the mean and standard deviation. The unpaired two-tailed t-test or one-way ANOVA using SPSS (version 19.0, USA) were used to determine whether there were any statistically significant differences between the means of the different treatment groups. Differences were considered significant when $p$ values $<0.05$.

\section{Results}

\section{Stability of bilateral sacroiliac joints}

Nine pelvic specimens were subjected to a force from 0 $\mathrm{N}$ to $400 \mathrm{~N}$, and displacement was measured using a sophisticated camera. Baseline displacement of M1, M2, and M3 was obtained prior to surgery. The results showed that displacement was represented mainly on the $Y$-axis (Fig. $3 \mathrm{a}-\mathrm{c}$ ). As the pelvic ring is fixed in the horizontal position, only vertical displacement is discussed in the following experiment. We used the relative vertical displacement (RVD) of M1 and M2 to represent the stability of the right sacroiliac joint, and the RVD of M2 and M3 to represent the stability of the left sacroiliac joint. The RVD of the right and left sides, before Salter pelvic osteotomy, were denoted by D1 and D2, respectively. D1 and D2 of the nine pelvic specimens were $0.63 \pm 0.19 \mathrm{~mm}$ and $0.54 \pm 0.24 \mathrm{~mm}$, respectively. There was no significant difference between D1 and D2 $(p=0.967)$. Unilateral Salter pelvic osteotomy was applied to nine pelvic specimens, and the RVD for the left and right sides was recorded. D3 and D4 represent the RVD of the operated and non-operated sides, 

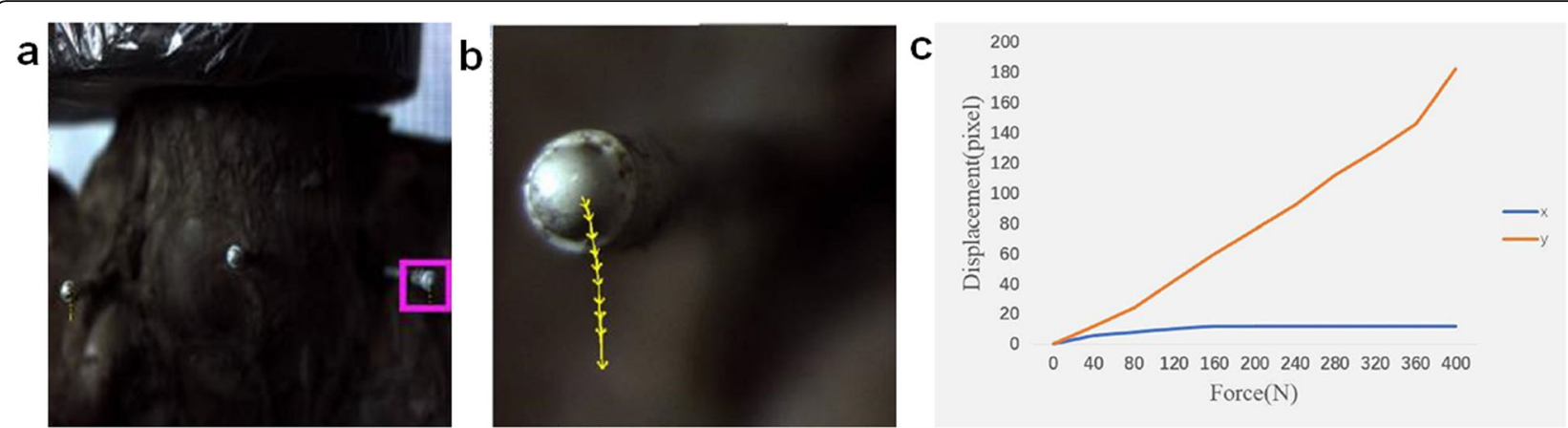

Fig. 3 The movement locus of marked point when a force added to the pelvis on the top of the fifth lumber from 0 to $400 \mathrm{~N}$. a The marked point in pink rectangle were analyzed. $\mathbf{b}$ The yellow line represents the movement locus of marked point in pink rectangle. $\mathbf{c}$ The blue and red line represents displacement of $X$-axis direction and $Y$-axis direction respectively when the force added from $0 \mathrm{~N}$ to $400 \mathrm{~N}$

respectively. D3 was $1.03 \pm 0.52 \mathrm{~mm}$, which was significantly lower than D4 $(2.07 \pm 0.74 \mathrm{~mm}, p<0.001)$. D3 was not significantly different compared with $\mathrm{D} 1(p=0.192)$ and D2 $(p=0.081)$ whereas D4 was significantly higher than both D1 and D2 $(p<0.001)$ (Fig. 4a). To assess whether one-stage bilateral Salter pelvic osteotomy affects pelvic stability, we chose six pelvic specimens for further (bilateral) osteotomy. D5 represents the RVD of the former (initial) osteotomy side and D6 represents the RVD of the latter (subsequent) osteotomy side. The value of D5 was $1.32 \pm 0.57 \mathrm{~mm}$, which is not significantly different from D6 at a value of $1.16 \pm 0.48 \mathrm{~mm}(p=0.997)$. The values of D1, D2, D3, and D4 of the six pelvic specimens were $0.62 \pm 0.22 \mathrm{~mm}, 0.46 \pm 0.23 \mathrm{~mm}, 0.85 \pm 0.38$ $\mathrm{mm}$, and $1.79 \pm 0.65 \mathrm{~mm}$, respectively. D5 was significantly higher than D1 and D2 $(p<0.05)$. D6 was also higher than D1 and D2 and the difference between D6 and D2 was significant. Both D5 and D6 were higher than D3 and lower than D4, but the differences were not significant $(p>0.05)$ (Fig. 4b).

\section{Local stability of operation area}

The RVD of L1 to L2, and R1 to R2 represent the local stability of the operation area. D7 and D8 represent the RVD of the former (initial) operation side and the latter (subsequent) operation side, respectively. Unilateral Salter pelvic osteotomy was applied to nine pelvic specimens and the value of D7 (unilateral) was $0.58 \pm$ $0.21 \mathrm{~mm}$. The one-stage bilateral Salter pelvic osteotomy was applied to six pelvic specimens. The values of D7 (bilateral) and D8 (bilateral) of these six pelvic specimens were $0.50 \pm 0.23 \mathrm{~mm}$, and $0.52 \pm 0.27 \mathrm{~mm}$, respectively. There was no significant difference between D7 (unilateral), D7 (bilateral), and D8 (bilateral) $(p=0.763)$ (Fig. 5a).

\section{Axial stiffness assessment}

The baseline axial stiffness was $188.11 \pm 55.41 \mathrm{~N} / \mathrm{mm}$ before Salter osteotomy. After unilateral Salter pelvic osteotomy, the axial stiffness was lower $(119.06 \pm 43.47$ $\mathrm{N} / \mathrm{mm}$ ). For one-stage bilateral Salter pelvic osteotomy
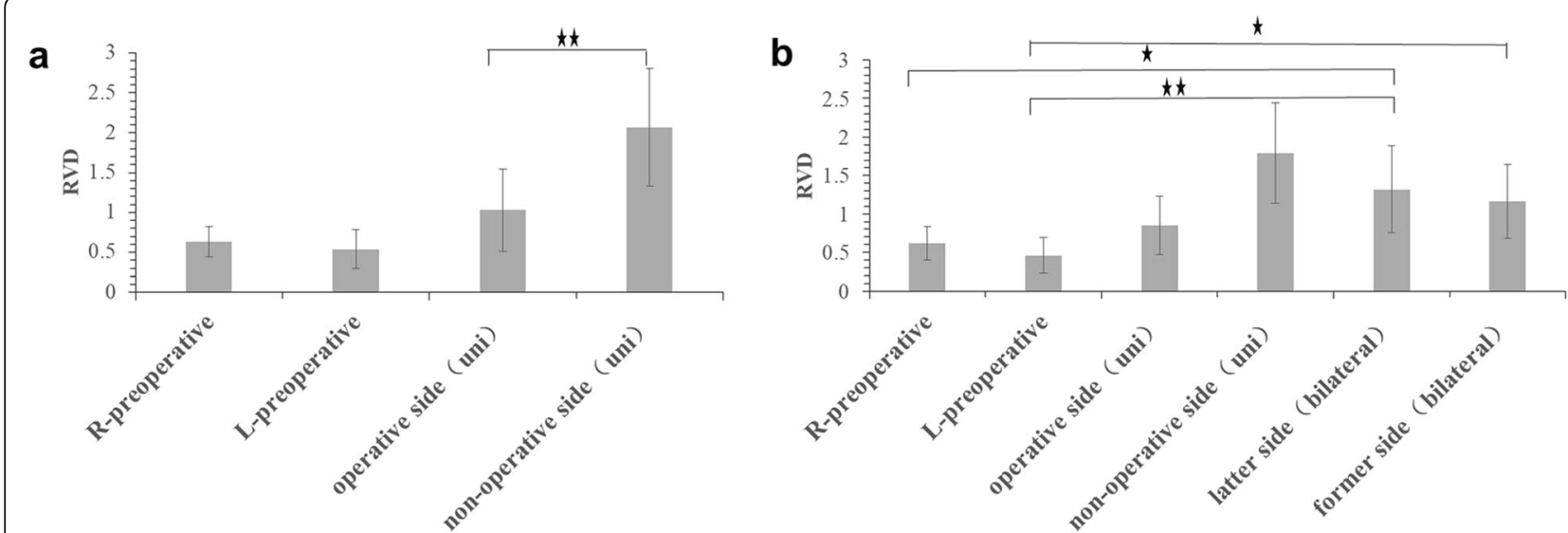

Fig. 4 Assessment of stability of bilateral sacroiliac joints. a Stability comparison of intact pelvis and pelvis treated with unilateral salter pelvic osteotomy. b Stability comparison among intact pelvis, pelvis treated with unilateral Salter pelvic osteotomy and one-stage bilateral Salter pelvic osteotomy $\left({ }^{\star} p<0.05,{ }^{\star \star} p<0.01\right)$ 
a

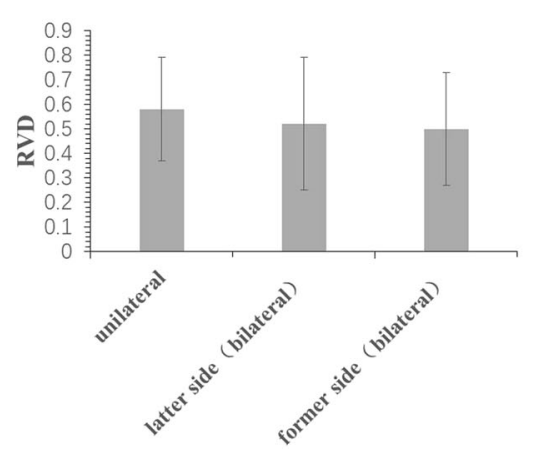

b

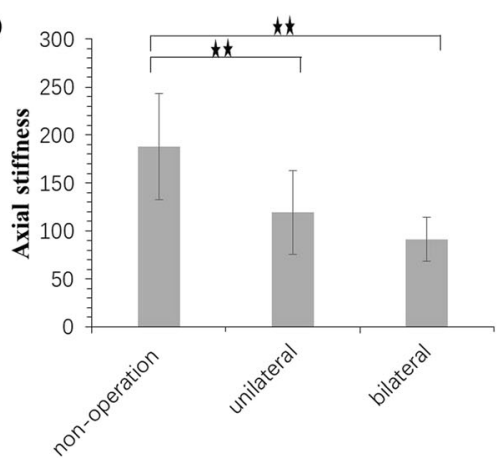

C

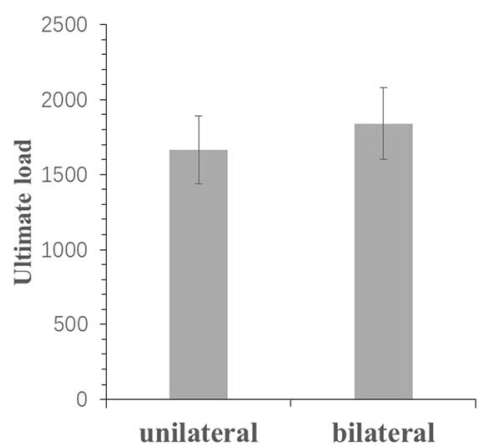

Fig. 5 Assessment of stability of local stability of operation area, ultimate load test and axial stiffness. a The RVD of local stability of operation area for different operation sites. b comparison of axial stiffness among non-operation group, unilateral salter pelvic osteotomy group and one-stage bilateral Salter innominate osteotomy group. c Comparison of ultimate load for unilateral salter pelvic osteotomy and one-stage bilateral Salter innominate osteotomy $\left({ }^{\star \star} p<0.01\right)$

specimens, the axial stiffness was $91.30 \pm 22.42 \mathrm{~N} / \mathrm{mm}$, which was lower than both the unilateral Salter pelvic osteotomy group and the non-operation group. There was a significant difference in axial stiffness between the non-operation group and both the unilateral Salter pelvic osteotomy group $(p=0.010)$, and the one-stage bilateral Salter pelvic osteotomy group $(p=0.001)$. However, there was no significant difference between the unilateral and one-stage bilateral Salter pelvic osteotomy groups $(p=0.480)$ (Fig. 5b).

\section{Ultimate load test}

The ultimate load test was applied to the unilateral and bilateral Salter pelvic osteotomy specimens. For the unilateral Salter pelvic osteotomy specimens, the ultimate load was $1664.27 \pm 226.26 \mathrm{~N}$ and $1840.83 \pm 241.55 \mathrm{~N}$ for the six one-stage bilateral Salter pelvic osteotomy specimens. There was no significant difference in the ultimate load between the two different treatment groups $(p=0.328)$ (Fig. 5c). All results of our research were also shown in Supplementary Table S1.

\section{Discussion}

DDH is a progressive disease with varying severity in children. The stability of the pelvic ring is mainly maintained by the posterior sacroiliac joint, the anterior pubic symphysis, and the surrounding ligaments, which tightly bind all bone blocks together to form the pelvic ring [13, 14]. In this study, nine child cadaver specimens were subjected to unilateral and six of those were subjected to one-stage bilateral Salter pelvic osteotomy. We found that one-stage bilateral Salter osteotomy had a similar effect on sacroiliac joint stability, local stability, axial stiffness, and ultimate load as unilateral Salter pelvic osteotomy.
The sacroiliac joint, and especially the surrounding ligaments, play an important role in the stability of the posterior pelvic ring. Salter pelvic osteotomy damaged the pelvic ring, but we found that the sacroiliac joint at the operation side after unilateral Salter pelvic osteotomy was more stable than at the non-operation side. This may be caused by a bilateral asymmetrical force. In Barnes's study, the researchers found that unilateral Salter pelvic osteotomy could lead to hip dysplasia on the healthy side [15]. We think that the pelvic stress on the operation side was transferred to the contralateral pelvic ring, as force hinge, carrying more loads which affected the stability of the non-operation side. However, one-stage bilateral Salter pelvic osteotomy cuts both the left and right ilium and both sides are fixed with the same treatment. This could lead to two hinge points making bilateral sacroiliac joint stress a symmetrical force.

Salter pelvic osteotomy cuts the ilium and is fixed with Kirschner wires. This study tested whether one-stage bilateral Salter pelvic osteotomy increases the risk of local stability. We found that the RVD of local stability for one-stage bilateral Salter pelvic osteotomy was between 0.50 and 0.52 $\mathrm{mm}$, which is lower than that for unilateral Salter pelvic osteotomy. Moreover, we considered that one-stage bilateral Salter pelvic osteotomy created a bilateral symmetrical force on both the left and right pelvic ring.

The axial stiffness of the pelvis reflects the displacement of the pelvis when subjected to axial pressure [16]. The greater the axial stiffness, the stronger the pelvic compressive capacity. We found that both unilateral and one-stage bilateral Salter pelvic osteotomy decreased the axial stiffness of the pelvis. In addition, this study showed axial stiffness of one-stage bilateral pelvic osteotomy $(91.30 \mathrm{~N} / \mathrm{mm})$ to be lower than that of unilateral Salter pelvic osteotomy $(119.06 \mathrm{~N} / \mathrm{mm})$ but the difference was not significant. The ultimate load reflects the 
stability of the whole pelvic ring, and we found that onestage bilateral Salter pelvic osteotomy did not decrease ultimate load, which verified the stability of one-stage bilateral Salter pelvic osteotomy. Interestingly, the ultimate load test showed that, regardless of whether unilateral or bilateral pelvic Salter osteotomy was performed, the pelvic ring was first destroyed at the sacroiliac joint, and not at the Kirschner wire fixation site. According to the current weight estimation formula "Weight $(\mathrm{kg})=$ 2 (age +4)" [17], the ultimate load of two Salter pelvic osteotomies is strong enough to support at least eight times the body weight of a child aged 6 years. All the results support the theory that one-stage bilateral pelvic Salter osteotomy is vertically stable. However, due to the preciousness of the cadaver specimens, the study did not test the ultimate load of a normal pelvic ring, so no comparison between a normal pelvic ring and a bilateral one-stage osteotomy pelvic ring was done in this study.

This study was the first to confirm the stability of onestage bilateral Salter pelvic osteotomy. Some surgeons have tried one-stage surgery for DDH disease and have followed up on efficacy. In Ochoa's study, 45 children suffering from congenital dislocation of the hip or acetabular dysplasia were assigned to one-stage bilateral Salter pelvic osteotomy (15 children) or successive Salter osteotomy (30 children). One-stage osteotomy was shown to be viable and had a better acetabular index and may be even better than that of a unilateral osteotomy [18]. In another study [19], a one-stage operation (using a Pemberton osteotomy for one hip and a Salter osteotomy for the other hip) was more economical and allowed more rapid recovery than a two-stage procedure comprising consecutive operations. In addition to Salter osteotomy, the stability of a one-stage Pemberton's pericapsular osteotomy, which also damages the pelvic ring, has been verified. In Zorer's study, 20 patients underwent a one-stage bilateral Pemberton's pericapsular osteotomy, and the results showed significant advantages over 2 separate consecutive interventions [20]. Another study by Agus' team evaluated the clinical outcome of 12 children (24 hips) who treated with one-stage bilateral Salter pelvic osteotomy and compared with 12 patients (12 hips) who received unilateral Salter pelvic osteotomy, and found one-stage bilateral Salter pelvic osteotomy did not increase the mortality and postoperative complications [21]. And only the blood transfusion volume was higher in one-stage bilateral Salter pelvic osteotomy group than that of unilateral osteotomy group (170 cc vs $100 \mathrm{cc}$ ), which may be because bilateral osteotomy group needs longer operation time.

Although the stability of a one-stage bilateral Salter pelvic osteotomy was verified in this study, there were some limitations. First, there were a limited number of cadaveric pelvic specimens, and only six underwent one-stage bilateral Salter pelvic osteotomy. Second, the cadaver specimens belong to kids without any DDH, our model could not completely simulate live patients. The weight of the upper body is mainly transmitted to the pelvic ring through the bones, especially the spine, but some tissues such as skin, fascia, and muscle of psoas, and gluteals also transmit pressure. Third, according to our clinical experience, children who undergo one-stage bilateral Salter pelvic osteotomy receive plaster fixation and are allowed to start some exercises in bed before pelvic osteotomy healing, whereas the specimens in the study were static and the early postoperative activities of children could not be simulated. Fourth, in Salter osteotomy, the fulcrum point is the pubic symphysis, we evaluated the RVD of the surgical site, but did not evaluate the anterior and lateral displacement of the acetabulum on the contralateral side. In addition, the pubic symphysis, as the force hinge of Salter osteotomy, was very meaningful to explore the stability difference between unilateral and bilateral salter osteotomy. In future research, we need to further explore and improve these deficiencies. Despite these limitations, some important structures were protected and showed that one-stage bilateral Salter pelvic osteotomy is viable and stability for bilateral DDH.

In summary, this study was based on the pelvis of children aged 2 to 6 years and simulated one-stage bilateral Salter pelvic osteotomy. Compared with unilateral Salter pelvic osteotomy, one-stage bilateral Salter pelvic osteotomy is viable and stability from the aspect of sacroiliac joint stability, local stability, ultimate load, and axial stiffness. However, this finding was based on experimental research. Clinical treatment of patients is more complicated and is affected by many factors, such as the physical condition of the patient, hospital infrastructure, anesthesia level, doctor's experience, and patient compliance. We must carefully choose treatment options. In addition, a larger number of specimens and long-term clinical follow-up are necessary in future.

\section{Supplementary information}

Supplementary information accompanies this paper at https://doi.org/10. 1186/s13018-020-01683-w.

Additional file 1: Tables S1 Table the detail results of sacroiliac joint stability, local stability, ultimate load, and axial stiffness.

\section{Abbreviations}

DDH: Developmental dysplasia of the hip; RVD: Relative vertical displacement

\section{Acknowledgements}

The authors would like to acknowledge e all the colleagues on the Department of Anatomy, West China of Medicine, Sichuan University. 


\section{Ethics approval consent to participate}

The cadavers in this study were obtained from the Department of Anatomy, West China of Medicine, Sichuan University. All procedures performed in studies involving human participants were in accordance with the ethical standards and law for protection of cadavers sampling of the Chinese Government and with the 1964 Helsinki Declaration and its later amendments or comparable ethical standards.

\section{Authors' contributions}

Lang Li, Xiaodong Yang, and Bo Song contributed equally to this work. LL, $X D Y$, and BS carried out the experiment. JJ and LY wrote the manuscript. XYT supervised the project. All authors read and approved the final manuscript.

\section{Funding}

The study was supported by International cooperation project of Sichuan provincial science and technology department (2019YFS0265 and 2020YFS0082), postdoctoral fund of Sichuan University (2019SCU12034), and postdoctoral fund of West China Hospital (2018HXBH076).

\section{Availability of data and materials}

The datasets used and/or analyzed during the current study are available from the corresponding author on reasonable request.

\section{Consent for publication}

Consent for publication of the data was obtained from the relatives of the donors before the start of the study.

\section{Competing interests}

The authors declare that they have no conflict of interest.

\section{Author details}

'Department of Pediatric Surgery, West China Hospital, Sichuan University, Chengdu 610041, Sichuan, China. ${ }^{2}$ Department of Pediatric Orthopaedic Ward 1, Children's Hospital of Chongqing Medical University, Chongaing 400014, China.

Received: 29 January 2020 Accepted: 28 April 2020

Published online: 11 May 2020

\section{References}

1. Tong SH, Eid MA, Chow W, To MK. Screening for developmental dysplasia of the hip in Hong Kong. J Orthopaedic Surg (Hong Kong). 2011;19(2):200-3.

2. Salter RB. Role of innominate osteotomy in the treatment of congenital dislocation and subluxation of the hip in the older child. J Bone Joint Surg Am. 1966:48(7):1413-39.

3. Umer M, Nawaz H, Kasi PM, Ahmed M, Ali SS. Outcome of triple procedure in older children with developmental dysplasia of hip (DDH). J Pak Med Assoc. 2007;57(12):591-5.

4. Danielsson L. Late-diagnosed DDH: a prospective 11-year follow-up of 71 consecutive patients (75 hips). Acta Orthop Scand. 2000;71(3):232-42.

5. El-Sayed M, Ahmed T, Fathy S, Zyton H. The effect of Dega acetabuloplasty and Salter innominate osteotomy on acetabular remodeling monitored by the acetabular index in walking DDH patients between 2 and 6 years of age: short- to middle-term follow-up. J Child Orthop. 2012;6(6):471-7.

6. Lin CJ, Lin YT, Lai KA. Intraoperative instability for developmental dysplasia of the hip in children 12 to 18 months of age as a guide to Salter osteotomy. J Pediatr Orthop. 2000;20(5):575-8.

7. Chang $\mathrm{CH}$, Yang WE, Kao HK, Lee WC, Shih CH, Kuo KN. Prognostic radiographic factors in developmental dysplasia of the hip following Salter osteotomy. J Pediatr Orthop B. 2015;24(1):31-4.

8. Schmidutz F, Roesner J, Niethammer TR, Paulus AC, Heimkes B, Weber P. Can Salter osteotomy correct late diagnosed hip dysplasia: a retrospective evaluation of 49 hips after 6.7 years? Orthop Traumatol Surg Res. 2018; 104(5):637-43.

9. Vandergugten S, Traore SY, Docquier PL. Risk factors for additional surgery after closed reduction of hip developmental dislocation. Acta Orthop Belg. 2016:82(4):787-96.

10. Chen $Q$, Deng $Y$, Fang B. Outcome of one-stage surgical treatment of developmental dysplasia of the hip in children from 1.5 to 6 years old. A retrospective study. Acta Orthop Belg. 2015;81(3):375-83.
11. Erturk C, Altay MA, Yarimpapuc R, Koruk I, Isikan UE. One-stage treatment of developmental dysplasia of the hip in untreated children from two to five years old. A comparative study. Acta Orthop Belg. 2011;77(4):464-71.

12. Qadir I, Ahmad S, Zaman AU, Khan CM, Ahmad S, Aziz A. One-stage hip reconstruction for developmental hip dysplasia in children over 8 years of age. Hip Pelvis. 2018;30(4):260-8.

13. Puhakka KB, Melsen F, Jurik AG, Boel LW, Vesterby A, Egund N. MR imaging of the normal sacroiliac joint with correlation to histology. Skelet Radiol. 2004;33(1):15-28.

14. Eichenseer PH, Sybert DR, Cotton JR. A finite element analysis of sacroiliac joint ligaments in response to different loading conditions. Spine (Phila Pa 1976). 2011;36(22):E1446-52.

15. Barnes JR, Thomas SR, Wedge J. Acetabular coverage after innominate osteotomy. J Pediatr Orthop. 2011;31(5):530-3.

16. Taylor KF, Rafiee B, Inoue N, McHale KA, Howard RS, Chao EY. Linear increase in axial stiffness of regenerate callus during limb lengthening. Clin Orthop Relat Res. 2005:435:239-44.

17. Luscombe MD, Owens BD, Burke D. Weight estimation in paediatrics: a comparison of the APLS formula and the formula 'Weight=3(age)+7'. Emerg Med J. 2011;28(7):590-3.

18. Ochoa O, Seringe R, Soudrie B, Zeller R. Salter's single-stage bilateral pelvic osteotomy. Revue de chirurgie orthopedique et reparatrice de l'appareil moteur. 1991;77(6):412-8.

19. Ezirmik N, Yildiz K. Advantages of single-stage surgical treatment with salter innominate osteotomy and Pemberton pericapsular osteotomy for developmental dysplasia of both hips. J Int Med Re. 2012:40(2):748-55.

20. Zorer G, Bagatur AE. Single-stage bilateral Pemberton's pericapsular osteotomy in bilateral developmental dysplasia of the hip. Acta Orthop Traumatol Turc. 2002;36(4):288-94.

21. Agus H, Bozoglan M, Kalenderer O, Kazimoglu C, Onvural B, Akan I. How are outcomes affected by performing a one-stage combined procedure simultaneously in bilateral developmental hip dysplasia? Int Orthop. 2014; 38(6):1219-24

\section{Publisher's Note}

Springer Nature remains neutral with regard to jurisdictional claims in published maps and institutional affiliations.
Ready to submit your research? Choose BMC and benefit from:

- fast, convenient online submission

- thorough peer review by experienced researchers in your field

- rapid publication on acceptance

- support for research data, including large and complex data types

- gold Open Access which fosters wider collaboration and increased citations

- maximum visibility for your research: over $100 \mathrm{M}$ website views per year

At $\mathrm{BMC}$, research is always in progress.

Learn more biomedcentral.com/submissions 